\title{
Les nouveaux programmes pour l'école primaire
}

Christine Muller

\section{OpenEdition}

\section{Journals}

Édition électronique

URL : http://journals.openedition.org/ries/3429

DOI : 10.4000/ries.3429

ISSN : 2261-4265

\section{Éditeur}

Centre international d'études pédagogiques

\section{Édition imprimée}

Date de publication : 1 décembre 1996

Pagination : 94-97

ISSN : 1254-4590

\section{Référence électronique}

Christine Muller, «Les nouveaux programmes pour l'école primaire », Revue internationale d'éducation de Sèvres [En ligne], 12 I 1996, mis en ligne le 01 octobre 2013, consulté le 01 mai 2019. URL : http:// journals.openedition.org/ries/3429; DOI : 10.4000/ries.3429 


\section{Les nouveaux programmes pour l'école primaire}

Christine Muller

Depuis Jules Ferry, l'école élémentaire a connu une dizaine de renouvellements complets de programmes d'enseignement. Les nouveaux programmes de l'école primaire, fixés par l'arrêté du 22 février 1995, définissent, dans les perspectives tracées par le Conseil national des programmes, les contenus de l'enseignement et les compétences que les élèves doivent désormais acquérir cycle par cycle. Définis nationalement après de larges concertations, ils s'imposent à tous comme le cadre de référence nécessaire à l'action de chaque enseignant. Cette conception de programmes nationaux pluriannuels souligne la nécessité, pour les équipes pédagogiques, d'établir des progressions et des programmations annuelles qui constituent, dans chaque école, le cœur des projets de cycle.

Les nouveaux programmes de l'école primaire ont été conçus pour répondre à une double exigence: d'une part, simplifier les précédents programmes de 1985 et les recentrer sur l'essentiel; d'autre part, mettre en cohérence les contenus et les progressions de l'enseignement à la fois avec l'organisation de l'école en trois cycles pluriannuels ${ }^{1}$ et avec les compétences de fin de cycle, établies et publiées en 1991.

Prendre en compte cette nouvelle organisation en trois cycles amenait, en effet, à fixer un cadre de référence pour l'école maternelle qui, jusque là, n'avait jamais eu de "programme» au sens plein du terme. A l'école élémentaire, la volonté de simplification se traduisait, dès la première rédaction du nouveau texte, par des allégements substantiels en sciences et technologie et en histoire et géographie. De plus, des évolutions ou des thèmes nouveaux s'étaient imposés, en dix ans, au sein des disciplines elles-mêmes : l'initiation à l'environnement, l'éducation à la santé, à la sécurité, l'approche d'une morale de la responsabilité...

En juin 1994, le Nouveau contrat pour l'école fixait un objectif de travail - « de nouveaux programmes sont élaborés à l'école primaire et au collège. Mis en cohérence avec les cycles, ils sont allégés et recentrés sur les savoirs essentiels. Ils sont d'une lecture aisée » (mesure $n^{\circ} 3$ ) - et il donnait une méthode : "les enseignants sont directement associés à l'élaboration des programmes » (mesure $\left.\mathrm{n}^{\circ} 136\right)$.

1 Décret $n^{\circ}$ 90-788 du 6 septembre 1990. 
Durant l'été 1994, est mis en chantier - avec le souci constant que le texte et la démarche soient lisibles et compréhensibles par tous - un projet de programmes, élaboré par la direction des Écoles en collaboration avec l'Inspection générale de l'Éducation nationale, des spécialistes et des praticiens enseignants. Ce projet tient compte, au niveau des horaires, de l'introduction d'études dirigées et de la généralisation de l'initiation aux langues vivantes dès le cours élémentaire.

\section{La consultation nationale des enseignants : une méthode qui a fait école}

Publié au Bulletin officiel du $1^{\mathrm{er}}$ septembre 1994 et soumis à la consultation nationale de tous les enseignants, le projet de programme est étudié et commenté au sein de chaque école, dans les conseils de maîtres, en liaison avec les inspecteurs de l'Éducation nationale; l'ensemble des instituteurs et des professeurs des écoles peuvent s'exprimer ainsi sur sa rédaction, son contenu et sur sa faisabilité.

Pour la première fois dans l'histoire de la République, un projet de programmes d'enseignement est directement communiqué pour avis à tous ceux qui ont pour mission de les mettre en œuvre. Même si les sceptiques manifestent un attentisme prudent, les maîtres apprécient le fait d'anticiper la lecture des programmes avant leur parution officielle, c'est-à-dire non seulement de s'y préparer, mais aussi, espèrent-ils, d'infléchir le projet dans le sens de l'allégement et du pragmatisme.

L'opération conduit effectivement la majorité des enseignants à mener une réflexion pédagogique de fond, intégrant les aspects de méthodologie et d'évaluation. Dans quelque trente mille écoles, en liaison avec les inspecteurs de l'Éducation nationale, les maîtres jugent de la cohérence des programmes par rapport aux objectifs de l'école primaire, tous peuvent s'exprimer sur leur rédaction, leur faisabilité, leur pertinence discipline par discipline.

\section{Les résultats}

Cette consultation nationale - innovation étendue ensuite au collège s'est traduite par des contributions d'une grande qualité. Plusieurs milliers de maîtres, individuellement ou à travers leurs équipes pédagogiques, transmettent leurs observations et leurs suggestions. Sont parvenus au ministère de l'Éducation nationale: plus de mille courriers personnels, souvent porteurs de signatures multiples, mille trois cents réponses Minitel et les synthèses départementales issues de la réflexion menée au sein des conseils de maîtres et élaborées au niveau de chaque inspection académique.

Expressions individuelles et synthèses collectives de l'enseignement public et de l'enseignement privé se trouvent validées et confirmées les unes par 
les autres à quelques très faibles nuances près. A la quasi-unanimité sont reconnues la bonne lisibilité et la pertinence des programmes par rapport aux compétences définies en 1991. Les objectifs et les contenus des programmes des trois cycles sont très largement approuvés, mais trois enseignants sur cinq sont réservés quant à leur faisabilité dans l'horaire imparti et dans les conditions actuelles de moyens et de formation. Les enseignants constatent, en effet, qu'il n'y a pas de véritable allégement des programmes dans la mesure où l'horaire de chaque discipline est diminué pour dégager le temps nécessaire à l'enseignement d'une langue vivante et aux études dirigées, ressenti comme du temps "perdu » pour les autres disciplines, notamment les mathématiques, l'histoire, la géographie et les sciences.

\section{Les amendements}

Les observations des enseignants sont prises en compte et aboutissent au rééquilibrage des horaires (surtout au profit des mathématiques), à des allégements significatifs (en mathématiques, grammaire, conjugaison, éducation civique, etc.) ou à des reformulations qui tendent toutes à privilégier la démarche concrète sur l'approche théorique. Les notions considérées comme prématurées ou mal situées à l'école élémentaire sont retardées d'un cycle au suivant ou reportées au collège.

Les ajouts de texte proviennent de précisions apportées pour limiter le champ ou atténuer la portée d'une étude, ou le niveau des exigences. Le souci est de rendre les contenus d'enseignement moins formels, moins théoriques et de préférer systématiquement l'entrée concrète dans les savoirs que l'enfant doit s'approprier : l'enfant doit apprivoiser progressivement les notions nouvelles à partir de sa propre expérience dans son environnement immédiat.

Les nouveaux programmes de l'école primaire affirment l'importance des apprentissages fondamentaux et donnent la priorité à la maîtrise de la langue française et à l'éducation civique. Ils préparent à l'entrée au collège en faisant émerger progressivement les disciplines d'enseignement de la scolarité secondaire. Ainsi au cycle des apprentissages fondamentaux, la rubrique « Découverte du monde » se différencie progressivement au cycle des approfondissements pour laisser l'histoire et la géographie, les sciences et la technologie acquérir leur véritable statut de discipline.

\section{Diffusion et application des programmes - Édition des manuels}

Le texte, dans sa nouvelle version, reçoit l'avis favorable du Conseil national des programmes et est approuvé par le Conseil supérieur de l'éducation le 8 décembre 1994. 
Publiés au Bulletin officiel ${ }^{2}$, les Programmes de l'école primaire sont adressés gratuitement en juin 1995 à tous les enseignants des écoles publiques et privées sous contrat ${ }^{3}$, augmentés d'une actualisation des tableaux de compétences à acquérir au cours de chaque cycle de l'école primaire, établis en 1991.

\section{Élaboration des programmes de l'école primaire : le calendrier des travaux}

- 1993-1994 : les nouveaux programmes sont mis au point en collaboration avec l'Inspection générale, des spécialistes, des enseignants...

- septembre 1994 : consultation nationale de tous les instituteurs sur un projet de texte publié au BO du $1^{\text {er }}$ septembre 1994 ;

- octobre-novembre 1994 : concertation avec les organisations représentatives (syndicats, associations de parents...) ;

- novembre 1994: remise en chantier du projet pour répondre à la demande générale de rééquilibrage des horaires et d'allégement ;

- décembre 1994 avis (favorable) du Conseil national des programmes, puis présentation (et approbation) au Conseil supérieur de l'éducation ;

- publication au BO spécial n 5 du 9 mars 1995.

Concernant la mise en œuvre des programmes, le maître a, dans sa classe, la responsabilité individuelle du choix des démarches prenant en compte la diversité des élèves et de leurs acquisitions effectives. D'où le développement d'une pratique d'évaluation régulière s'appuyant naturellement sur l'opération d'évaluation nationale mise en place en CE2 et en $6^{\mathrm{e}}$, mais aussi sur la banque d'outils élaborés par la direction de l'Évaluation et de la Prospective et sur l'usage d'outils d'évaluation internes à l'école et à la classe.

\section{L'application des programmes de 1995 : un calendrier sur trois ans}

Septembre 1995 : maternelle, cours préparatoire (CP), cours élémentaire $2^{\mathrm{e}}$ année (CE2) ;

Septembre 1996 : cours élémentaire $1^{\text {re }}$ année (CE1), cours moyen $1^{\text {re }}$ année (CM1) ;

Septembre 1997 : cours moyen $2^{\mathrm{e}}$ année (CM2).

2 B.O. spécial $n^{\circ} 5$ du 9 mars 1995.

3 Édition CNDP, Savoir-Livre (tirage 550000 exemplaires). 
Une mise en œuvre progressive sur trois ans vise à étaler le renouvellement - et leur financement par les municipalités - des manuels scolaires dans les écoles. Les éditeurs scolaires, en effet, mettent à jour les ouvrages en fonction des modifications de contenus d'enseignement. Le choix des manuels scolaires relève, dans chaque école, du conseil des maîtres qui doit informer le conseil d'école (où sont représentés le maire et les parents d'élèves), des principes qui ont présidé à leur choix. Les services du ministère de l'Éducation nationale ne sont pas habilités à porter un avis ou agrément sur les ouvrages publiés par des éditeurs privés. Néanmoins, les inspecteurs de l'Éducation nationale, qui contrôlent les activités conduites dans les classes, sont amenés à donner des conseils aux enseignants sur les démarches pédagogiques à adopter et à attirer leur attention sur le contenu des manuels scolaires.

\title{
DOCUMENT
}

\author{
UN APERCQU DU CONTENU \\ DES PROGRAMMES DE L'ÉCOLE PRIMAIRE (1995)
}

\section{Cycle des apprentissages premiers}

La maternelle est une école, un lieu d'éducation avec des objectifs précis, un lieu d'apprentissages défini dans le cadre d'un programme - pour la première fois la maternelle a un programme - traduit en cinq domaines d'activités mais sans emploi du temps imposé.

Les enfants apprennent à :

- vivre ensemble, partager avec d'autres des activités et des espaces communs, nouer des relations avec les adultes et avec leurs camarades, communiquer, découvrir des règles de la vie collective ;

- parler et construire leur langage, s'initier au monde de l'écrit, lier l'expérience au langage, maîtriser le langage oral et le confronter à l'écrit, prendre conscience de ce que c'est que lire et écrire, découvrir le fonctionnement de l'écrit et la culture écrite ;

- agir dans le monde, explorer l'espace, acquérir des compétences sensorielles, "mettre en jeu " leur corps selon des conduites réglées dans des environnements particuliers (eau, neige...);

- découvrir le monde, des objets, de la matière, du vivant, de l'espace, de l'image, être sensible aux problèmes d'hygiène, de consommation, de sécurité ;

- imaginer, sentir, créer, développer leur sensibilité, leur imagination, leur créativité, leur expression au contact de la musique, des arts plastiques, de la danse, du théâtre... 
Les enfants enrichissent leurs connaissances dans les situations qui les amènent à : dessiner, écrire, classer, sérier, dénombrer, mesurer, reconnaître des formes, se repérer dans l'espace, dans le temps et, à travers toutes ces activités, à se construire des instruments pour apprendre.

\section{Cycle des apprentissages fondamentaux}

Le cycle des apprentissages fondamentaux (grande section de maternelle, CP et CE1) met l'accent sur l'acquisition des langages - français et mathématiques - et des repères que donnent l'éducation civique et le champ des disciplines relatives à la découverte du monde; il fait une place importante au développement de la motricité et de la sensibilité.

La maîtrise de la langue française

L'oral: prendre la parole, écouter les autres, décrire, raconter, inventer des histoires, réciter des textes poétiques.

La lecture : il n'y a pas de méthode imposée, chaque maître choisit la plus appropriée pour que les élèves parviennent à enrichir le capital de mots, saisir les relations entre l'oral et l'écrit, percevoir l'organisation générale des phrases et du texte, se familiariser avec les textes et s'adapter à des formes variées, lire à haute voix, lire silencieusement, écouter le maître lire.

L'écriture : acquérir une graphie lisible, soigner la présentation d'un cahier, composer de petits textes d'abord dictés à l'adulte, puis rédigés de façon de plus en plus autonome, distinguer les différentes fonctions d'un écrit: expression d'une idée, d'un sentiment, communication d'un message, mise en mémoire d'une information... jouer sur les mots, les structures, les images, la langue poétique.

\section{Mathématiques}

Objectif : structurer les connaissances et développer l'aptitude à la recherche et au raisonnement. A partir de problèmes simples et en explorant des méthodes, des notions nouvelles, découvrir les nombres (jusqu'à 1000 ) et la numération décimale, maîtriser la technique de l'addition, aborder celle de la multiplication et de la soustraction, reconnaître quelques figures géométriques, repérer, reproduire, construire, mesurer...

La découverte du monde

A la maternelle, déjà, les enfants commencent à "découvrir le monde ». La découverte du monde n'est pas une nouvelle matière, c'est une manière d'approcher différents domaines de connaissance, des espaces et des époques plus ou moins proches, des phénomènes du monde de la matière et du monde du vivant. L'élève observe, compare et classe, manipule, construit, élabore de petits projets techniques, utilise un vocabulaire de plus en plus précis pour en parler, développe son goût de l'invention, son sens de l'innovation. 


\section{L'éducation civique}

Une pratique réfléchie de la vie en commun et de la vie de l'école donne les repères sociaux indispensables.

\section{L'éducation artistique}

Objectifs : développer sa sensibilité, sa capacité de création, découvrir des œuvres d'art, approcher des démarches d'artistes.

Deux disciplines artistiques obligatoires :

- l'éducation musicale: activités vocales, activités corporelles et instrumentales, activités d'écoute ;

- les arts plastiques.

Ouverture sur d'autres domaines artistiques: théâtre, expression dramatique, danse, images...

L'éducation physique et sportive

- développement de la motricité : courir, sauter, lancer, nager...

- action adaptée en fonction d'un effort, d'un risque à apprécier, de règles à observer individuellement ou collectivement.

\section{Le cycle des approfondissements}

Le cycle des approfondissements (CE2, CM1, CM2) poursuit les apprentissages du cycle précédent et introduit les premiers concepts et les démarches propres aux disciplines qui structureront la scolarité au collège (exemple, le champ disciplinaire découverte du monde, abordé aux deux cycles précédents se scinde en disciplines à part entière: l'histoire et la géographie, les sciences et la technologie.

Progressivement, les élèves assimilent les méthodes de travail propres à chaque discipline qui leur permettront d'acquérir une autonomie suffisante pour l'entrée au collège.

Les compétences transversales précédemment abordées sont approfondies : méthodes de travail, utilisation d'une documentation...

L'éducation à la santé, à la consommation, à la sécurité permettent de mettre en pratique des règles de vie à l'école en dehors de l'école.

A partir de leur environnement, les élèves acquièrent des connaissances et des compétences qui leur permettront de comprendre le monde dans lequel ils vivent.

Ils acquièrent ainsi les fondements d'une démarche scientifique : raisonner, poser des questions et proposer des solutions; de savoir-faire plus techniques: concevoir, fabriquer, transformer; enfin concernant l'informatique, ils utilisent l'ordinateur de façon raisonnée et au service des apprentissages de disciplines variées : maîtrise de la langue, sciences...

L'éducation artistique (éducation musicale et arts plastiques) a un rôle important : production, invention, observation et écoute pour développer l'imagination créatrice et acquérir une formation culturelle.

L'éducation physique et sportive contribue à l'équilibre des rythmes de vie de l'enfant. 